\title{
An Australian Aboriginal birth cohort: a unique resource for a life course study of an Indigenous population. A study protocol Susan M Sayers*1, Dorothy Mackerras ${ }^{1}$, Gurmeet Singh ${ }^{1}$, Ingrid Bucens ${ }^{2}$, Kathryn Flynn ${ }^{1}$ and Alison Reid ${ }^{3}$
}

\begin{abstract}
Address: ${ }^{1}$ Public Health and Chronic Disease Division, Menzies School of Health Research Darwin, Northern Territory, Australia, ${ }^{2}$ World Health Organisation, Dili, East Timor and ${ }^{3}$ School of Population Health, University of Western Australia, Perth, Australia

Email: Susan M Sayers* - sue@menzies.edu.au; Dorothy Mackerras - dorothy@menzies.edu.au; Gurmeet Singh - gurmeet@menzies.edu.au; Ingrid Bucens - ingbucens@hotmail.com; Kathryn Flynn - pharlap@octa4.net.au; Alison Reid - alisonr@dph.uwa.edu.au

* Corresponding author
\end{abstract}

Published: 6 March 2003

BMC International Health and Human Rights 2003, 3:1

This article is available from: http://www.biomedcentral.com/l472-698X/3/I

(c) 2003 Sayers et al; licensee BioMed Central Ltd. This is an Open Access article: verbatim copying and redistribution of this article are permitted in all media for any purpose, provided this notice is preserved along with the article's original URL.
Received: 12 December 2002
Accepted: 6 March 2003

\begin{abstract}
Background: The global rise of Type 2 diabetes and its complications has drawn attention to the burden of non-communicable diseases on populations undergoing epidemiological transition. The life course approach of a birth cohort has the potential to increase our understanding of the development of these chronic diseases. In 1987 we sought to establish an Australian Indigenous birth cohort to be used as a resource for descriptive and analytical studies with particular attention on non-communicable diseases. The focus of this report is the methodology of recruiting and following-up an Aboriginal birth cohort of mobile subjects belonging to diverse cultural and language groups living in a large sparsely populated area in the Top End of the Northern Territory of Australia.

Methods: A prospective longitudinal study of Aboriginal singletons born at the Royal Darwin Hospital 1987-1990, with second wave cross-sectional follow-up examination of subjects 19982001 in over 70 different locations. A multiphase protocol was used to locate and collect data on 686 subjects with different approaches for urban and rural children. Manual chart audits, faxes to remote communities, death registries and a full time subject locator with past experience of Aboriginal communities were all used.

Discussion: The successful recruitment of 686 Indigenous subjects followed up 14 years later with vital status determined for $95 \%$ of subjects and examination of $86 \%$ shows an Indigenous birth cohort can be established in an environment with geographic, cultural and climatic challenges. The high rates of recruitment and follow up indicate there were effective strategies of follow-up in a supportive population.
\end{abstract}

\section{Background}

The global rise in rates of Type 2 diabetes and its complications has drawn attention to the burden of non-communicable diseases on populations undergoing epidemiological transition [1,2].
The Australian Aboriginal population has features consistent with being at an early stage of epidemiological transition. In the Northern Territory, the rate of low birth weight (13\%) for Aboriginal Australians is twice that of non-Aboriginal Australians [3]. Aboriginal children have high rates of infectious diseases and infant malnutrition 
$[4,5]$. At the same time Aboriginal adults have excessive rates of obesity and chronic non-communicable diseases with life expectancies 10-15 years less than the non-Aboriginal Australians [6]. This premature adult mortality is largely due to respiratory, cardiovascular and renal disease, independently or as a complication of type 2 diabetes [7]. The natural histories of these diseases, the role of maternal health and whether poor intra-uterine growth contributes to these outcomes are still largely unknown.

The life course approach of a birth cohort study has the potential to assist in understanding these issues [8]. Prospective longitudinal studies have already been established in a number of developed populations [9-12] but only a few studies relate to populations undergoing epidemiological transition [13-15]. There are perceived difficulties and complexities in conducting studies within the Australian Aboriginal populations because of their diverse cultural and linguistic groups. Currently we know of no other prospective studies in this group.

In 1987 we sought to establish a prospective longitudinal Aboriginal birth cohort with the initial hypotheses relating to the influences of birth antecedents and perinatal outcomes on childhood growth and hospital admissions. With the increasing international interest on the fetal and childhood antecedents to adult disease, these aims were later extended to examine hypotheses relating birth antecedents and perinatal outcomes to potential risk factors for chronic diseases in childhood.

The focus of this report is the methodology of establishing and locating an Indigenous birth cohort of mobile subjects belonging to diverse cultural groups living in a large sparsely populated area with poor transport and communication infrastructures [16]. Only selected features which demonstrate the unique character of the cohort will be reported. Detailed results will be published in subsequent publications.

\section{Methods \\ Design}

A prospective longitudinal study of a birth cohort.

\section{Subjects}

Aboriginal children living in the Top End of the Northern Territory of Australia.

\section{Setting}

The Northern Territory (NT) is the most sparsely populated jurisdiction in Australia and is traditionally divided into two at the 20th latitude south. The "Top End" of the NT is 11 degrees from the equator at its northern point and includes the urban capital Darwin. The Royal Darwin Hospital (RDH) is the only government hospital for the capital and the surrounding geographic Darwin Health Region (DHR) of 120,000 square kilometres (similar in area to England). But the hospital also functions as a tertiary referral centre for the adjacent health regions of the Top End and northern Western Australia an area of over 2 million square kilometres.

The Top End has a marked seasonal variation with a Dry season of almost no rain, and a two month "build up" with frequent intense electrical storms leading to a Wet season. The four month Wet season of monsoon rain with cyclones leads to limitation of year round road and aircraft access to many communities [16].

In the Top End the indigenous population is found in the suburbs of Darwin, in seasonal camps around the city, in small towns and in remote isolated rural communities and outstations.

Many families in isolated communities have no communication infrastructure, some are limited to a single solar powered public phone, and in many communities there are no public phones in working order [16].

Within the Indigenous population there is a high level of intra-regional mobility [17] and at least 30 Aboriginal languages and dialects are in daily use [18] The children have more than one name and change their names for prolonged periods, many have dual house status within a community and between communities and school attendance is infrequent and sporadic [19].

The only health delivery in the rural areas is conducted through government community health clinics staffed with permanent nursing staff and Aboriginal health workers. Few clinics have daily access to a doctor and most have access on a weekly, fortnightly or monthly basis [20].

\section{First wave at birth: 1987-1990 \\ Preparation}

Community consultation about the proposed study was undertaken at meetings with council members of the bigger Aboriginal communities close to Darwin and by phone with the leaders of more distant Aboriginal communities. Letters of endorsement were obtained from high profile Aboriginal leaders and elders. Aboriginal health and educational workers from rural communities were informed about the study when attending courses in Darwin.

\section{Eligibility}

Babies were eligible for enrolment in the study if they were live born singletons delivered at the RDH between January 1987 and March 1990 to a mother who was recorded as Aboriginal in the Delivery Suite Register. There 


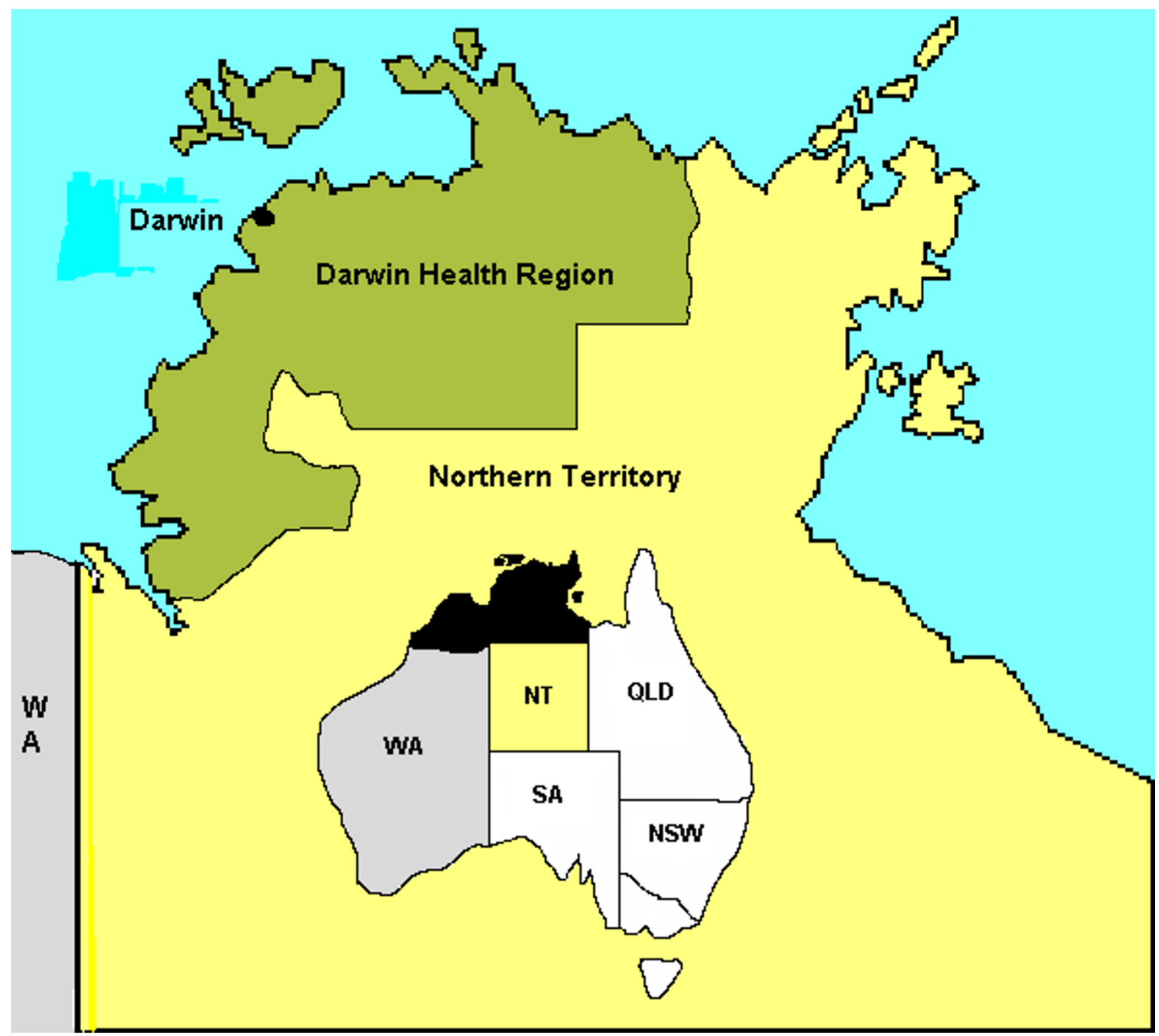

Figure I

Northern Territory map with Darwin Health Region and Australia insert with area for referrals to the tertiary centre. Darwin: Situation of the Royal Darwin Hospital receiving all admissions from the Darwin Health Region (green in Northern Territory map) and high risk referrals from adjacent health regions in the Northern Territory and Western Australian (black on the Australian insert).

were no exclusions. Of the 1238 eligible Aboriginal babies the majority (1053) were routine referrals from the local health region. Within this region at that time $90 \%$ of pregnant Aboriginal mothers come to the RDH to deliver their babies [21]. The remaining babies were high risk referrals transferred 'in utero' from adjacent NT health regions
(13.9\%) and a few (1.5\%) were from across the Western Australian border (Figure 1).

\section{Process}

Soon after birth the Aboriginal research assistant explained the broad outline of the proposed study to prospective participants. If agreement was obtained the 


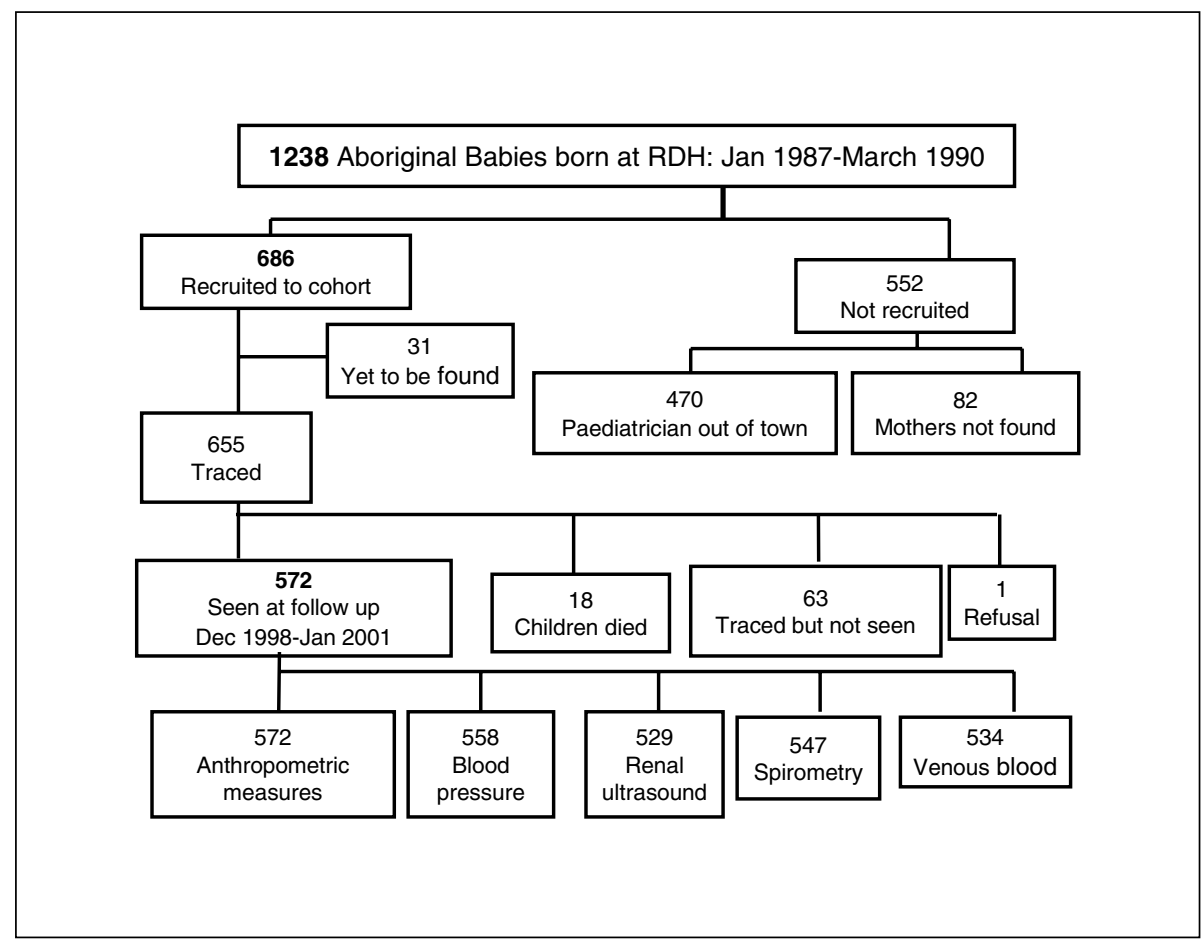

\section{Figure 2}

Flow chart of Aboriginal Birth Cohort I987-200I.

neonatal paediatrician (SS) visited the Aboriginal mothers within four days of delivery. The paediatrician discussed the aims of the study in detail, and obtained permission to recruit the baby into the cohort. An interview was conducted and an examination of the baby undertaken, including an estimation of gestational age. Plans to see the baby again in ten years were discussed.

\section{Response}

Recruitment was dependent primarily on the whether the neonatal paediatrician was in town. She was intermittently absent for a total of 5 months during this time. Recruitment was also dependent on whether mothers could be found in the hospital surroundings as they often absented themselves for extended periods of time (Figure 2). All the mothers found and interviewed agreed to participate.
During the recruitment period, 686 Aboriginal maternal child pairs were enrolled into the study, accounting for $55 \%$ of potential recruits.

Although the subjects were not randomly selected, for the whole cohort there were no significant differences in mean birth weight, or gender ratio between those recruited and not recruited using the unpaired test (Table 1). For the DHR subset of babies the birth weight frequencies were also similar for those recruited and not recruited (Figure 3).

\section{Data collection}

Table 2 includes the list of data collected at wave 1 and the source of the information. 


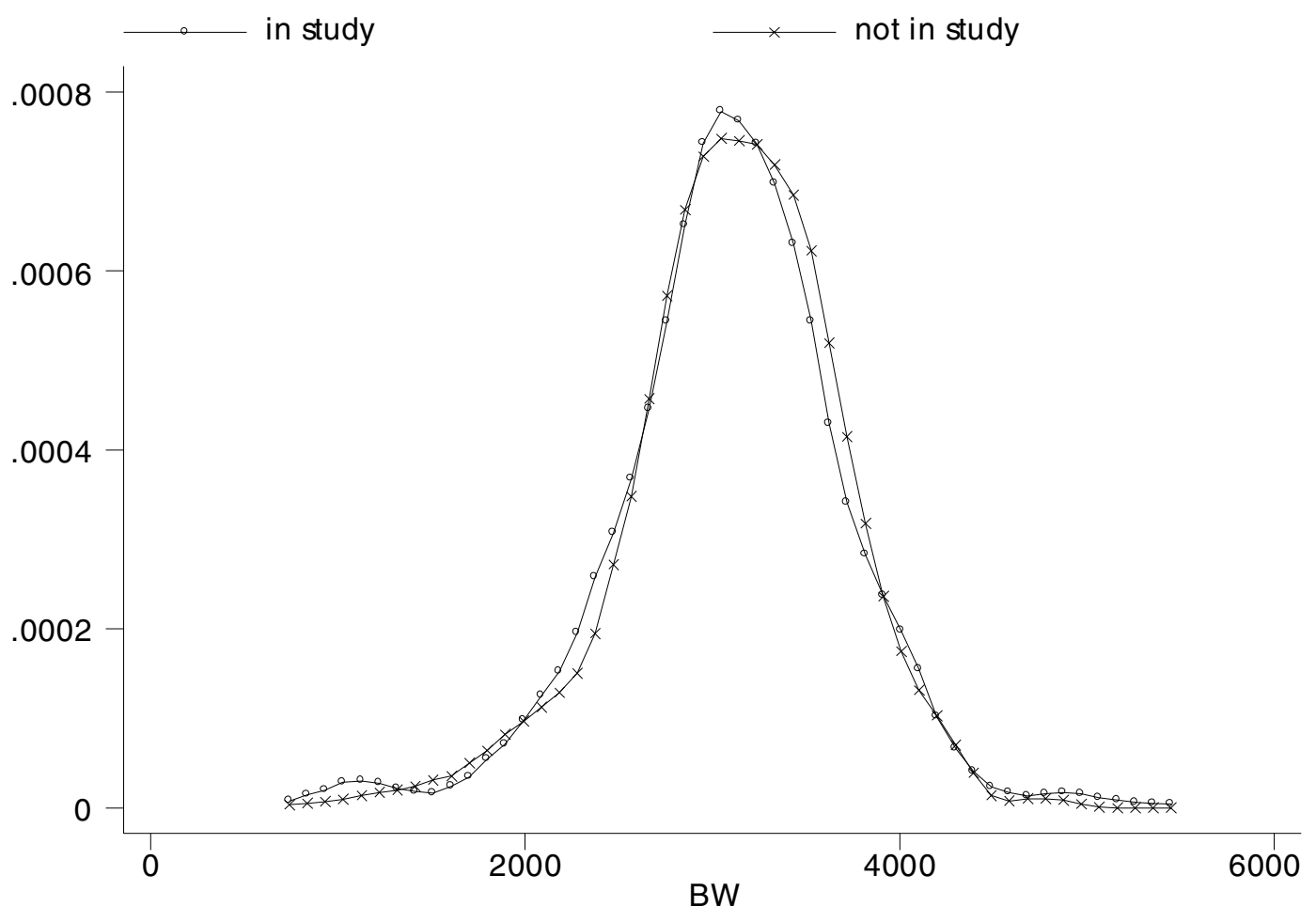

Figure 3

Birth weight frequencies for babies recruited and not recruited at birth into Aboriginal Birth Cohort I9872001 .

Table I: Characteristics of those recruited and not recruited at birth. Aboriginal Birth Cohort Study $1987-1990$

\begin{tabular}{lcccc}
\hline & Total births & Recruited & Not recruited & P value \\
\hline Number & 1238 & 686 & 552 & \\
Mean birth weight gms (sd) & $3042(635)$ & $3020(668)$ & $3068(592)$ & 0.2 \\
Male n (\%) & $643(52)$ & $352(55)$ & $291(45)$ & 0.6 \\
\hline
\end{tabular}

Identifying features recorded at time of recruitment

The child's birth name, date of birth, hospital record number, birth weight and the mothers name and address were all recorded at the time of delivery. For urban mothers, the addresses were a street number and suburb. For all others the addresses consisted of the Aboriginal commu- nity, locality of camp or outstation that the mother had come from at the time of delivery

\section{Wave I ethical considerations}

The study was approved by the Joint Institutional Ethics Committee of the Royal Darwin Hospital and the Menzies School of Health Research (JIEC). In the post-natal wards 
Table 2: Data collected and source of information and investigators involved for phases I and 2. Aboriginal birth cohort study

\begin{tabular}{|c|c|c|}
\hline Time interval & Data collected & Source of information and investigator responsible \\
\hline \multirow[t]{6}{*}{$1987-1990$} & Date of birth & Medical records a \\
\hline & Sex & Physical examination a \\
\hline & Birth weight, length & Medical records a \\
\hline & Head circumference & Physical examination a \\
\hline & Gestational age [25] & Physical examination a \\
\hline & Placental weight & Medical records a \\
\hline \multirow[t]{28}{*}{$|998-200|$} & Anthropometric and nutritional & \\
\hline & Date of assessment & \\
\hline & Height & Portable stadiometer $b$ \\
\hline & Weight & Tannita model TBF 52/ b \\
\hline & Head, mid upper arm, waist circumferences [29] & Physical examination $\mathrm{b}$ \\
\hline & Sub scapular and triceps skin folds [29] & Harpenden calipers b \\
\hline & Puberty grade $[30,3 I]$ & Physical Examination e \\
\hline & Respiratory & \\
\hline & Respiratory function [32] & Vitalograph 2120 spirometer ${ }^{c}$ \\
\hline & Respiratory symptoms and signs & Physical examination $\mathrm{c}$ \\
\hline & Hospital admissions with respiratory diagnosis & Medical records c \\
\hline & Renal & \\
\hline & Renal ultrasound [33] & Ultrasonography c \\
\hline & Random urinary albumen creatinine ratio & Beckman image $\mathrm{h}$ \\
\hline & Random urinalysis & Urinary dipstick $f$ \\
\hline & Metabolic and cardiovascular & \\
\hline & Liver ultrasound & Ultrasonography c \\
\hline & $\begin{array}{l}\text { Fasting plasma glucose, total cholesterol, HDL-C, LDL-C, } \\
\text { triglycerides }\end{array}$ & Hitachi 917 Autoanalyser, Roche reagents h \\
\hline & Apolipoprotein A-I, Apolipoprotein B, lipoprotein(a) & BN2-Behrring $g$ \\
\hline & Fasting plasma insulin & Immunoassay, AIA-PACK g \\
\hline & Red blood cell folate & BayerAC I80, Bayer reagents $h$ \\
\hline & Blood pressure & Welch Allyn Lifesigns monitor ${ }^{f}$ \\
\hline & Haematological and infection & \\
\hline & Full blood count & Coulter Max $\mathrm{M}^{\mathrm{h}}$ \\
\hline & Impetigo, scabies, ear discharge, nasal discharge & Physical examination e \\
\hline & Social & \\
\hline & Individual, Household items & Child/carer interview ${ }^{f}$ \\
\hline & Community items $[34,35]$ & Data base examination $\mathrm{i}$ \\
\hline \multirow[t]{2}{*}{$|987-200|$} & Longitudinal growth data & \\
\hline & Weight, length, head circumference & Health records ${ }^{f}$ Examination $i$ \\
\hline
\end{tabular}

a Neonatal paediatrician SS. b Nutritional epidemiologist DM. c Paediatrician IB. d Paediatrician GS. e All team paediatricians. $f$ All team members. g Perth laboratory. $h$ Darwin laboratory. i Aboriginal research assistant. j Team biostatistician AR

after the Aboriginal research assistant explained the study to the mother the chief investigator made sure the mother understood about the study and recorded maternal verbal permission to enrol the baby in the cohort (now verbal consent is no longer acceptable to ethics committees).

\section{Cohort subject details}

The birth outcomes for the total cohort are listed in Table 3 . For the geographically defined DHR subset (570), the mean birth weight was 3083 grams (SD 600 grams), 13\% were low birth weight (LBW): birth weight $<2500$ grams, $7 \%$ preterm: $<37$ weeks gestation and $25 \%$ were small for gestational age (SGA): weight $<10$ th percentile of weight for gestational age using a Melbourne based reference standard comparable in time with the cohort recruitment $[22,23]$.

The babies from outside the DHR had a lower mean birth weight and higher percentages of LBW, preterm and SGA births (Table 3 ) due to this subset being the selected high risk 'in utero' referrals from adjacent health regions.

\section{Interim period}

At approximately four years of age, names, current primary carer and current address were checked on 320 children. By the age of four years, $30 \%$ of children who were traced had changed the name they commonly used once and $18 \%$ had changed their address once. Two percent $(2 \%)$ of children had had three different names and $2.4 \%$ 
Table 3: Perinatal outcomes. Aboriginal Birth Cohort Study 1987-1990

\begin{tabular}{lccc}
\hline Perinatal outcome & DHR subset (570) & High risk referred subset (II6) & Total cohort (686) \\
\hline Mean birth weight gms (sd) & $3098 \mathrm{~g}(60 \mathrm{I})$ & $2639(830)$ & $3020 \mathrm{~g}(668)$ \\
LBW n (\%) & $76(13)$ & $46(40)$ & $122(18)$ \\
Mean gestational age wks (sd) & $38.9(1.72)$ & $37.7(2.63)$ & $38.7(1.95)$ \\
SGA n (\%) & $126(25)$ & $4 \mathrm{I}(4 \mathrm{I})$ & $167(24)$ \\
Preterm n (\%) & $37(7)$ & $26(26)$ & $63(10)$ \\
Mean PI (sd) & $2.60(0.26)$ & $2.48(0.28)$ & $2.58(0.26)$ \\
\hline
\end{tabular}

had had four different addresses. Immediately prior to the second wave a manual audit of all hospital records was undertaken to check for changes in identifying features on the last known admission.

\section{Wave 2 follow-up 1998-200I}

Follow-up commenced in December 1998 and continued till January 2001. The follow-up team consisted of the original neonatal paediatrician (SS), two other paediatricians (GS, IB), a nutritional epidemiologist (DM) and a full-time research assistant (KF) who had past experience of remote communities and was both a nurse and commercial aircraft pilot.

\section{Tracing first phase}

For the rural children, key personnel with an in depth knowledge of the local community were identified at the community health clinic and school nearest to the last known address. A list of the children with the latest identifying information was faxed to these key rural workers with a request for return faxes supplying important new information (e.g. maternal death, name and address change) and confirmation of the child's current presence in that area.

For urban children, the last known address was contacted by phone or letter followed by a personal visit by KF if these were unsuccessful

Hospital admissions to the RDH, limited to cohort names and next of kin, were checked daily.

\section{Tracing second phase}

For rural children, information from the local workers, relatives, next of kin, friends and other cohort families was obtained when visiting the last known community.

For urban children information was gathered from the next of kin, neighbours, school rolls, health clinics and hospital records.
Tracing third phase

In addition to novel and opportunistic approaches (e.g. attending local football games) the above procedures were repeated at 3-6 month intervals till all leads were exhausted.

\section{Data collection}

For rural children preparations for a visit to a targeted area were commenced approximately 2-3 months in advance (see Figure 4). Communities were visited on a week day about the middle of the school term. The team worked predominantly in spaces within the local school area but other buildings, verandas, private homes and health clinic rooms were all used. Local Aboriginal people acted as interpreters, advocates and tracers. For communities with a large number of cohort subjects KF visited prior to the team's arrival and we entered into a formal agreement with a local Aboriginal person to work as a temporary research assistant.

For urban children preparations for a group to be examined commenced about 2 weeks in advance. The children were seen on a Saturday morning at the clinic of the RDH and transport was provided for the mother or carer to and from the clinic. Some children were seen in private homes, local school rooms and community clinic rooms.

Table 2 includes the list of data collected at wave 2 and the source of the information. As the procedures took about 45 minutes to complete, jigsaw puzzles and a photo album of other cohort subjects undergoing procedures were provided to keep the children occupied and informed. At the end children were given juice, muffins and fruit.

The venepuncture was always done 30 minutes after the application of a local anaesthetic cream. The majority of blood samples were centrifuged and separated within 4 hours of collection. From remote communities they were taken to routine aircraft routes and transported to the Darwin laboratory packed in Styroform containers with cold bricks. 
Children: present, current names and caregivers, mother still alive Permits: valid permit for entry to Aboriginal lands for all team members, Permissions: to visit community from council, school and health clinic. Consents: informed written consent from parents Access: road passable, need for 4WD, fuel type and outlets, river tide height and times, plane times, availability of charter, air time for trip, airstrip location, transport from airstrip Team needs: accommodation and food availability Study needs: space, power points, centrifuge, refrigeration, sample transport, availability of Aboriginal helpers Community activities: pay day, other health visitors, school holidays, school activities

Cultural issues: ceremony, death

Day before: check weather forecast, check community activities

\section{Figure 4}

Response at follow-up

At follow-up the vital status was determined for $95 \%$ of the original cohort with an $86 \%$ follow-up rate of living children. (Figure 1). Weather and access difficulties meant 63 traced children could not be examined. Mortality found by our investigations and possible mortality in untraced children was checked by examination of hospital records and registers at the Northern Territory and Western Australian Birth Death and Marriages Offices. From this, one untraced child was found to have died in Western Australia. Hence of the original 686 children 5\% remain untraced.

For those children from the DHR subset there were no significant differences between those seen and those not seen or found Table 4 . For the total cohort, only the mean gestational ages of 38.9 and 38.4 weeks were significantly dif- ferent between those children seen and those children not seen or found.

At follow up 18 children were known to have died including 10 neonatal deaths and 5 traumatic deaths, one nonaccidental. The children known to have died had a shorter gestation and lower mean birth weight reflecting the neonatal deaths due to preterm births.

At follow up, 27 mothers were reported to have died. Inspection of 25 available death certificates showed 11 due to chronic non-communicable disease and 5 due to trauma, including three non-accidental deaths.

\section{Wave 2 ethical considerations}

Permission to follow-up and take biological samples from the cohort was sought and granted in a new application to the JIEC. This application included a teleconference with 
Table 4: Characteristics of children according to seen and not seen status at time of follow up. Aboriginal Birth Cohort Study I998-200 I

\begin{tabular}{|c|c|c|c|c|c|}
\hline \multirow[b]{2}{*}{ Characteristic } & \multicolumn{2}{|c|}{ Total Cohort } & \multicolumn{2}{|c|}{ DHR Subset } & \multirow[b]{2}{*}{ Died before follow-up } \\
\hline & Seen at follow up & Not seen or found & Seen at follow-up & Not seen or found & \\
\hline Number & 572 & 96 & 489 & 71 & 18 \\
\hline Mean birth weight gms (sd)a & $305 I(630)$ & $2983(719)$ & $3112(578$ & $3127(607)$ & $2229(1015)$ \\
\hline $\begin{array}{l}\text { Mean gestational age } \\
\text { weeks }^{\mathrm{a}}\end{array}$ & $38.9(1.78)$ & $38.4(1.72)^{*}$ & $39(1.61)$ & $38.8(1.47)$ & $35.4(5.9)$ \\
\hline$\%$ male $^{b}$ & $52 \%$ & $48 \%$ & $52 \%$ & $54 \%$ & $39 \%$ \\
\hline$\%$ Low birth weight ${ }^{b}$ & 16 & 22 & 12 & 14 & \\
\hline$\%$ Preterm $^{b}$ & 10 & 14 & 7 & 9 & \\
\hline$\%$ Small for gestational age ${ }^{b}$ & 27 & 28 & 25 & 25 & \\
\hline
\end{tabular}

$a t$ test $b$ Fisher exact test $* \mathrm{p}=<0.05$ for difference between children seen and not seen or found at follow-up

Table 5: Cross-sectional characteristics at follow-up. Aboriginal Birth Cohort Study 1998-200 I

\begin{tabular}{lccc}
\hline Characteristic & DHR subset & Referred in utero subset & Total cohort \\
\hline Seen at follow-up n (\%) & $489(86)$ & $83(86)$ & $572(86)$ \\
Mean age years (sd) & $11.4(1.1)$ & $11.9(1.15)$ & $11.5(1.1)$ \\
$\%$ commenced puberty & 45 & 54 & 46 \\
\hline
\end{tabular}

an Aboriginal sub-committee of the JIEC. Permission to store biological samples was not obtained.

Two communities required formal presentation of the proposed protocols to their respective medical boards and field work did not commence until permission had been granted from the board. Both these communities requested access to the information collected.

In the field an information sheet modified by the Aboriginal sub-committee was given to the primary carer and explained by local Aboriginal helpers with aid of the photo album. Written permission was obtained from the primary carer. Verbal permission for each procedure was obtained from the children with the right of refusal emphasised. Thirty eight children (6\%) refused to have their blood taken and a few children refused less invasive procedures. Verbal consent to take pictures for a photo album of children undergoing different procedures was obtained from the primary carer and the child.

For rural children, the results of the examinations were sent to the nearest health clinic with suggested actions for those with abnormal results. For large rural communities the Chairman of the council was given a general report of the overall findings for that community.

For urban children, results were sent to the primary carer and/or to a health worker, doctor or clinic of their choice.

\section{Characteristics of subjects at follow-up}

At follow-up for the routine subset, the mean age was 11.4 years and $48.5 \%$ had commenced puberty (Tanner stage $1)$.

$21 \%$ of children were suburban residents of Darwin, $70 \%$ were rural residents and 9\% were living in "other" locations which included two small country towns as well as Aboriginal communities and camps in and around Darwin city. At the time of follow-up a third of children living in the city had moved from a rural locality whereas only $3 \%$ of rural children were originally from the city The children who had been referred 'in utero' from outside the DHR were seen later in the follow-up and hence the high risk survivors at the time of follow-up were older, taller and a greater number had commenced puberty (Table 5).

Pertinent to the follow-up, only $25 \%$ of children spoke English exclusively in the family home, $22 \%$ did not have a parent as the primary carer and 39\% lived in communities that did not have road access throughout the year (not including the islands).

\section{Costs}

Separate funding was obtained for each wave.

Costs of follow-up were dependent on the distance travelled to a locality, the number of potential children to see and the number of children actually seen per visit. Transport costs were reduced by team members travelling in se- 
Table 6: Estimated minimal costs per child for 5 team members visiting a remote community for 4 days to see a potential 40 subjects and seeing 30

\begin{tabular}{lc}
\hline Item & Estimated cost Aus $\$$ \\
\hline Aircraft travel $\$ 160 \times 5$ team members & 800 \\
Accommodation $\$ 30$ per night $\times 3$ nights $\times 5$ & 450 \\
Subsistence allowance $\$ 60$ per night $\times 3$ nights $\times 5$ & 900 \\
Local vehicle hire $\$ 20$ per day $\times 4$ days & 80 \\
Fuel $\$ 25$ per day $\times 4$ days & 100 \\
Aboriginal helper 8 hour day $\times 3$ days & 640 \\
Wages for full time research assistant & 740 \\
Food for per child $\$ 6 \times 40$ children & 240 \\
Total for 30 children & 3950 \\
\hline Cost per child & 131 \\
\hline
\end{tabular}

ries to smaller communities by hitchhiking rides on routine medical flights. On occasions aircraft were chartered for extensive round trips and private vehicles were frequently used to access communities close to Darwin.

Table 6 presents the estimated minimal cost per child incurred for an average 4 day team visit to a larger remote community with 40 potential cohort subjects to examine These costs do not include those tracing the children, preparations for the visit and time of the part time investigators. Catchment of children per trip varied considerably but averaged approximately $65 \%$ of potential children per visit. With each succeeding visit the cost per child increased. When small numbers were reached the team split up to travel in series. The substantial cost of seeing a single very isolated child accounts for the 63 children who were traced but not seen.

\section{Discussion}

This longitudinal prospective birth cohort is a unique resource for both descriptive and analytical studies relating to an Indigenous population undergoing epidemiological transition. It demonstrates that Indigenous cohort studies can be undertaken in challenging geographic and cultural circumstances.

Although $70 \%$ of pregnant mothers came from the rural localities, with features of a traditional lifestyle, they all came to the RDH for delivery where we performed careful measurement and recording of the size of their baby.

Few mothers knew their last menstrual period date or had an early dating fetal ultrasound (6\% and $12 \%$ respectively) This is similar to other traditional populations [24]. Gestational age at delivery was therefore ascertained by the neonatal pediatrician (SS) using the Dubowitz scoring system, always within the recommended four days post- delivery [25]. This system of 23 post-natal neurological and physical criteria has been validated for other populations $[26,27]$ and has been found to be a satisfactory method for the estimation of gestational age in Aboriginal neonates [28]. These gestational estimations allowed accurate identification of the preterm and small for gestational age babies.

The mothers were not randomly selected, but there was no evidence of any selection bias between those babies seen and not seen in regard to gender ratios, mean birth weights and birth weight frequencies. Aboriginality was based on maternal self-identification and hence those subjects with an Aboriginal father and non-Aboriginal mother are not represented.

There were significant geographic challenges in locating and ascertaining data from the subjects in childhood. The children lived in approximately 70 different locations over a vast area outside Darwin city with unsealed roads of variable quality subject to seasonal flooding. Transport costs were kept to a minimum by making use of spare seats on routine aircraft flights and occasionally using the team pilot with chartered planes Great flexibility was required to allow for lack of daylight flying hours, cyclone warnings and other adverse weather conditions.

The cohort population was characterized by diverse language groups, a high level of family mobility and name changes. In the $75 \%$ of families with English as a second language, local helpers/interpreters answered questions and went over the information sheet with the help of the photo album. A list of the outstanding names was presented at each rural community. Even when subjects were known to be in a community flexibility of time was needed, as children frequently moved from house to house. The children had multiple names relating to kinship, 
clans and relationships within family groups. Commonly name changes occur following the death of a namesake. Aggressive exploring of each name was necessary. Of the identifying features recorded at birth the most reliable was the unique hospital record number used in all health services of the Northern Territory. Relating different names back to this number in the health clinic frequently proved an identity. A digital photo record (not available at the time) of the mother and baby at the time of delivery and a record of all the names including the Aboriginal name given at birth would have been useful additions to the identifying records.

Rural children were more likely to be found in the "off week" of the fortnightly welfare payment, in the middle of the week, in the middle of the school term. But events within a community such as ceremonies, deaths, funerals and the conduct of large community meetings affected the availability and willingness of subjects to participate in the study. Team flexibility was essential to accommodate unexpected community events.

Nevertheless, despite these challenges, an $86 \%$ follow-up rate for the total cohort indicates the study team's perseverance was successful. For the DHR subset, there were no significant differences between those children seen at follow-up and those not seen or found in regard to mean birth weight, gestational age and the proportions of low birth weight, preterm and small for gestational babies. This lack of attrition bias for the DHR subset means the children belonging to this subset are likely to be a representative sample of the current peri-pubertal population of the region.

The high follow-up rate also shows the study population was a co-operative one willing to take part in a long term study. All the rural Aboriginal communities were interested in the study and we were able to negotiate well at the community level to enable us to find more children. Some mothers were pleased and surprised to see the principal investigator again, and many were glad their child was being checked. Other mothers wanted their children to become part of the study. Most children seemed to enjoy the attention and blood taking was not difficult with the help of local anaesthetic cream. We were encouraged by the acceptability of this study and now view this cohort as a resource for a life long study. Future waves broadening the scope of hypotheses to encompass psycho-social and educational aspects are planned.

This longitudinal study was undertaken in an Australian Indigenous population and is most applicable to this population. Nevertheless parts of the methodology may be generalised to other indigenous cohort studies. The success of this follow-up of an Indigenous birth cohort may encourage other investigators exploring causal pathways of chronic adult disease in indigenous populations.

\section{Competing interests}

None declared.

\section{Authors' contributions}

SS conceived of the study, carried out the first wave, participated in the second wave and drafted the manuscript.

DM was responsible for growth and nutritional assessment in the second wave and helped with the manuscript. GS was responsible for the renal assessment in the second wave and helped with the manuscript and production of figures. IB was responsible for the respiratory assessment in the second wave and helped with the manuscript. KF coordinated the second wave, traced all the subjects, and liased with all Aboriginal. communities and government agencies. AR is responsible for data management and analysis and helped with the manuscript.

\section{Acknowledgements}

This work was supported by the National Health and Medical Research Council of Australia, the Colonial Foundation Trust, and the Channel 7 Research Foundation of SA inc. We thank Alan Ruben for advice on form and content of the manuscript and especially thank the Aboriginal mothers and children who agreed to be part of this study.

\section{References}

I. Amos AF, McCarty DJ and Zimmet $P$ The rising burden of diabetes and its complication: estimations and projections to the year 20 I 0. Diabet Med I997, I4(Suppl 5):SI-85

2. Yusif S, Reddy S, Ounpuu S and Anand S Global burden of cardiovascular diseases. Part I: General considerations, the epidemiologic transition, risk factors, and impact of urbanization. Circulation 2001, I04:2746-2753

3. Nassar $\mathrm{N}$ and Sullivan EA Australia's mothers and babies 1999. Perinatal Statistics Series No I I. Australian Institute of Health and Welfare. National Perinatal Statistics Unit Sydney 200I,

4. Currie BJ and Brewster DR Review article. Childhood infections in the tropical north of Australia. J Paediatr Child Health 200I, 37:326-330

5. Ruben $A$ and Walker $A$ Malnutrition among rural Aboriginal children in the Top End of the Northern Territory. Med J Aust 1995, 1 62:400-403

6. Hoy WE, Norman RJ, Hayhurst BG and Pugsley DJ A health profile of adults in a Northern Territory Aboriginal community, with emphasis on preventable morbidities. Aust NZ J Public Health 1997, 2 I(2): I $21-126$

7. Parades $Y$ and Cunningham J Placing Aboriginal and Torres Strait Islander mortality in an international context. Aust NZ J Public Health 2002, 26(I): I I-16

8. Kuh D and Ben-Shlomo $Y$ An introduction: life course approach to aetiology of adult chronic disease. In: A life course approach to chronic disease epidemiology (Edited by: Kuh D, Ben-Shlomo Y) Oxford, Oxford University press 1997, 3-15

9. Golding J Children of the nineties. A longitudunal study of pregnancy and childhood based on the population of Avon (ALSPAC). WE Med J 1990, I 05(3):80-82

10. European longitudinal study of pregnancy and childhood (ELSPAC) [http://www.ich.bris.ac.uk/ELSPAC/protocol/default.htm]

II. National longitudinal survey of children and youth (Canada) [http://www.hrdc-drhc.gc.ca/sp-ps/arb-dgra/nlscy-elnej/home.shtml] 
12. Silva PA The Dunedin multidisciplinary health and developmental study: a 15 year longitudinal study. Paediatr Perinat Epidemiol 1990, 4:76-107

13. Yajnek C Interactions and perturbations in intrauterine growth and growth during childhood on the risk of adult onset disease. Proc Nutr Soc 2000, 59:257-265

14. Adair LS Filipino children exhibit catch-up growth from age 2 to 12 years. J Nutr 1999, I 29(6): I I 40- I I 48

15. Crowther NJ, Cameron N, Trusler J and Gray IP Association between poor glucose tolerance and rapid post-natal weight gain in seven year old children. Diabetologia 1998, 4I: I 163-I I67

16. Bailie R, Siciliano F, Dane G, Bevan L, Paradies Y and Carson B Communications and Transport In: Atlas of health-related infrastructure in discrete Indigenous communities. Northern Territory Australia: Cooperative Research Centre for Aboriginal and Tropical Health 200 I, 4I-44

17. Martin $D$ and Taylor J Ethnographic perspectives on the enumeration of Aboriginal people in remote Australia. JAPA 1996 |3(I):|7-3|

18. Black P Aboriginal languages in the Northern Territory. School of Australian Linguistics. Darwin. Darwin Community College 1983.

19. Northern Territory Department of Education Attendance and participation In: Learning lessons. An independent review of Indigenous education in the Northern Territory. Darwin, Government Printing Office of the Northern Territory 1999, I4I-I54

20. Bailie R, Siciliano F, Dane G, Bevan L, Paradies $Y$ and Carson B Health facilities and personnel In: Atlas of health-related infrastructure in discrete Indigenous communities. Northern Territory Australia: Cooperative Research Centre for Aboriginal and Tropical Health 200I, 49-66

21. Northern Territory Department of Health and Community Services Annual Report 199//1992 Darwin, Government Printer of the Northern Territory 1992, 125

22. World Health Organisation Definitions. International Classification of Diseases 10th Edition I: I235 Geneva; WHO 1993

23. Guaran R, Wein P, Sheedy M, Walstab J and Bleischer NA (1994) Update of growth percentiles for infants born in an Australian population. Aust NZ Obstet Gynaecol 1994, 34(I):39-49

24. Villar J and Belizan JM The relative contribution of prematurity and foetal growth retardation in developing and developed societies. Am J Obstet Gynecol 1982, 43:793-798

25. Dubowitz LMS and Dubowitz $\vee$ A clinical manual: gestational age of newborns Philippines, Addison-Wesley Publishing Company Inc 1977,

26. Breutin MJ Gestational age assessment in Nigerian born infants. Arch Dis Child 1973, 48:318-320

27. Singer $B$ and Wolfdorf J Estimation of gestational age of African newborn infants by a scoring system. S Afr Med J 1973, 47:20742077

28. Sayers SM and Powers JR An evaluation of three methods used to assess the gestational age of Aboriginal neonates. J Paediatr Child Health 1992, 28:312-317

29. Gibson RS Nutritional asessment. A laboratory manual. New York Oxford University Press 1993 .

30. Marshall WA and Tanner JM Variations in the pattern of pubertal changes in boys. Arch Dis Child 1970, 239: 1 3-23

31. Marshall WA and Tanner JM Variations in pattern of pubertal changes in girls. Arch Dis Child. 1969, 235:291-303

32. American Thoracic Society Standardisation of Spirometry 1994 Update. Am J Respir Crit Care Med 1995, I 52: I 107-36

33. Dinkel E, Ertel EM, Dittrich M, Peters H, Berres M and SchuilteWissermann $\mathrm{H}$ Kidney size in childhood: sonographic charts for kidney length and volume. Pediatr Radiol 1985, I 5:38-43

34. Australian Bureau of Statistics Regional Statistics Northern Territory Commonwealth of Australia 200I,

35. Bailie R, Siciliano F, Dane G, Bevan L, Paradies $Y$ and Carson B Health facilities and personnel In: Atlas of health-related infrastructure in discrete Indigenous communities. Northern Territory Australia: Cooperative Research Centre for Aboriginal and Tropical health 200I,

\section{Pre-publication history}

The pre-publication history for this paper can be accessed here:

http://www.biomedcentral.com/1472-698X/3/1/prepub

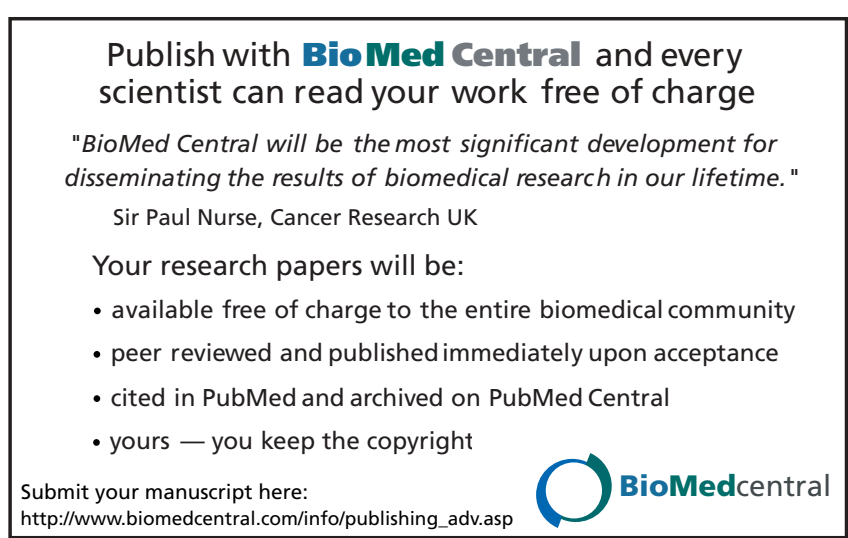

